\title{
Revisiting the Transverse Cervical Artery and Vein for Complex Head and Neck Reconstruction
}

\author{
Eitan Prisman, MD, MA, FRCSC ${ }^{1}$ Peter Baxter, MD ${ }^{2}$ Eric M. Genden, MD, MHCA, FACS ${ }^{2}$ \\ ${ }^{1}$ Division of Otolaryngology-Head \& Neck Surgery, Department of \\ Surgery, University of British Columbia, Vancouver, British \\ Columbia, Canada \\ 2 Department of Otolaryngology-Head \& Neck Surgery, ICAHN School \\ of Medicine at Mount Sinai School, New York, New York \\ Address for correspondence Eitan Prisman, MD, MA, FRCSC, Division \\ of Otolaryngology, Vancouver General Hospital, University of British \\ Columbia, 4th Floor, 2775 Laurel Street, Vancouver, B.C. Canada V5Z \\ $1 \mathrm{M9}$ (e-mail: eitan.prisman@ubc.ca).
}

J Reconstr Microsurg Open 2019;4:e54-e57.

\begin{abstract}
Keywords

- transverse cervical artery

- microvascular reconstruction

- head and neck reconstruction

Background Chemoradiotherapy is the primary treatment modality for glottic and pharyngeal subsites. Management of recurrence or second primaries in this setting is a surgical challenge requiring complex free flap reconstruction. One of the major barriers to effective reconstruction is the availability of suitable recipient vessels. We propose that the transverse cervical artery (TCA) is a viable option for complex head and neck reconstruction.

Methods A retrospective chart review of 230 consecutive free tissue reconstructive cases was performed by the senior author (EG).

Results Forty cases were identified that used the TCA for arterial anastomosis. Twenty-six patients had prior treatment, 13 of which had multimodality treatment. There were no microvasculature free flap failures and 5 minor flap complications. Conclusions Our experience with the TCA suggests it is a viable option for complex head and neck reconstruction, particularly in the setting of prior comprehensive neck dissection or radiation. In addition, the location of the TCA provides favorable pedicle geometry for microvascular anastomosis.
\end{abstract}

Recent advances in the treatment of head and neck cancer have been accompanied by an overall improved survival. While surgery followed by adjuvant radiation or chemoradiotherapy remains the primary treatment modality for oral cavity carcinoma, the advances in radiation techniques and chemotherapeutic agents have supported chemoradiotherapy as a common primary treatment modality for glottic and pharyngeal subsites. Nevertheless, a significant rate of recurrent disease or second primaries is a growing concern, particularly in the setting of field cancerization from previous exposure to known carcinogens. ${ }^{1}$ Surgical management of patients previously treated with surgery, chemoradiotherapy, or a combination thereof is common and often requires complex microvascular reconstruction to reconstitute form and function of ablative defects. In this setting, the reconstructive surgeon is faced with the challenge of

received

May 11, 2018

accepted after revision

January 19, 2019
DOI https://doi.org/

10.1055/s-0039-1692973.

ISSN 2377-0813. finding a suitable artery and vein to revascularize a free tissue transfer. The most frequently utilized vessels are branches from the external carotid and internal jugular system. These vessels are chosen based on the following parameters: location of the ablative defect, length of the pedicle, available vasculature, and the overall vascular geometry. However, patients previously treated with surgery often have these vessels compromised. Furthermore, previously radiated vessels are associated with an increased wall thickness and intimal dehiscence compared with nonirradiated vessels and may be associated with a higher risk of vascular thrombosis. ${ }^{2}$

The transverse cervical artery (TCA) is a branch of the thyrocervical trunk and is unique in that it is often outside the high-dose radiation target volume, particularly for primary malignancy in the oral cavity and oropharynx. Its
Copyright $\odot 2019$ by Thieme Medical Publishers, Inc., 333 Seventh Avenue, New York, NY 10001, USA. Tel: +1(212) 584-4662.
License terms

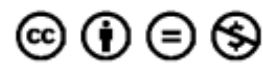


anatomical position in the inferior neck results in it being partly shielded by the clavicles in terms of radiation effects. Furthermore, surgical sacrifice of the TCA is less common, especially in the absence of an aggressive level IV neck dissection. While many microvascular surgeons utilize the transverse cervical vessels, there is a scarcity of literature looking at specifically addressing the TCA system in previously treated patients. Previous reports utilizing the TCA include a series of six and nine patients with encouraging results in a vessel depleted neck. ${ }^{3,4}$ Herein, we present a retrospective review of 230 consecutive head and neck free flap reconstruction to identify the utilization of the TCA and discuss the technique of, and advantages for, using the TCA for complex reconstructions.

\section{Methods}

Following institutional ethical review board, a retrospective chart review of all free tissue reconstructive cases from Jan 2012 to Jan 2014 by the senior author was performed to identify reconstructions using the TCA. Operative reports were reviewed, and cases were included only when the TCA was specified as the anastomotic vessel. Clinical charts were then reviewed for relevant clinical characteristics including age, stage of disease, defect type, free flap used for reconstruction, prior treatment modalities, complications, and length of hospital stay.

\section{Results}

Of the 230 microvasculature reconstruction cases reviewed, 40 cases (27 male, mean age of 65.1 years and 13 females, mean age 55.5 years) were identified that used the TCA for arterial anastomosis. The right TCA was used in 30 cases, where the venous anastomosis was performed with the right transverse cervical vein (TCV) and the right external jugular vein in 24 and 6 of the cases, respectively. The left TCA was used in 10 cases where the left TCV and left external jugular vein were used in 8 and 2 of the cases, respectively. Twentyeight patients had prior treatment, including 4 with surgery alone, 9 with radiation \pm chemotherapy, and 15 with surgery and radiation \pm chemotherapy. There were no microvasculature free flap failures and only 5 minor flap complications including two salivary fistulas, one hematoma, one partial flap loss, and one wound infection.

Ninety percent of patients had microvascular reconstruction for oncological ablative defects including squamous cell carcinoma as the most common (80\%) followed by salivary gland malignancies (5\%), sarcoma (2.5\%), and basal cell carcinoma (2.5\%). The oral cavity made up $44 \%$ of the ablative defects and the rest a combination of pharyngeal (25\%), cutaneous (20\%), or laryngeal (18\%) defects. The choice of free flap reconstruction included the anterior lateral thigh (50\%), radial forearm (37.5\%), fibular (7.5\%), and rectus abdominis flap (5\%).

There were no major complications related to the use of the TCA. Five patients had minor complications including one necrosis of a portion of the flap, one hematoma, one wound breakdown, and two salivary fistulas. Two patients were readmitted following discharge for hypercalcemia from over supplementation, and one patient required a prolonged hospital stay for psychiatric management. The average length of hospital stay was 9.5 days, typical of patients recovering from microvascular reconstruction.

\section{Discussion}

\section{TCA Anatomy}

The TCA is most commonly a branch of the thyrocervical trunk but may branch directly from the subclavian artery or internal mammary artery in 17 and $2 \%$ of cases, respectively. ${ }^{5}$ The artery courses in a posterior and lateral direction toward the trapezius and passes deep to the omohyoid muscle and superficial to the scalene muscles and brachial plexus (-Fig. 1). Just lateral to the omohyoid it gives off the supraclavicular artery. The TCA then divides into two branches before reaching the trapezius, the superficial branch which supplies the upper third of the trapezius muscle, as well as the deep branch which nourishes the middle third of the trapezius. ${ }^{6}$ If the TCA arises directly from the subclavian, it tends to run posteriorly and may pass through the upper and middle trunks of the brachial plexus. ${ }^{7}$ The TCV course is more variable than the artery in relationship to the omohyoid. The vein may run deep to the omohyoid in $75 \%$ of patients and superficial in approximately $25 \%$ of patients. ${ }^{8}$ The TCV can drain to either the external jugular vein or subclavian vein. When the TCV is not suitable for microvascular anastomosis due to vessel size discrepancy, then the external jugular vein is a great option. The external jugular vein exits over the posterior aspect of the sternocleidomastoid muscle (SCM) in level V of the neck and is in close proximity to be used as a substitute; its caliber in this location is 3 to $5 \mathrm{~mm}$ (-Fig. 1).

\section{TCA Technique}

While others have recommended the use of a Doppler ultrasound to locate the TCA, in our experience the vessel can be exposed with careful dissection in the triangle that is composed by the posterior border of the SCM, the superior border of the clavicle, and the superior portion of the omohyoid muscle. This can be approached either through the neck dissection incision or from a separate parallel horizontal incision overlying the midportion of this anatomical triangle. Unless the TCA arises directly from the subclavian artery, the brachial plexus should not be exposed, and the plane of dissection should be limited to the loose fat pad overlying the scalene muscle within which the vessels are located. ${ }^{9}$ The phrenic nerve will lie deep to the fascia overlying the anterior scalene and should be deep to the dissection plane. The phrenic nerve is often visualized but the fascia overlying it and the scalene muscles should not be disturbed. Care should be taken when identifying the branching pattern of the TCA so that it is not confused for the vertebral artery with obvious consequences. Exploration of the left TCA should be performed carefully to prevent inadvertent injury to the thoracic duct; however, this should 


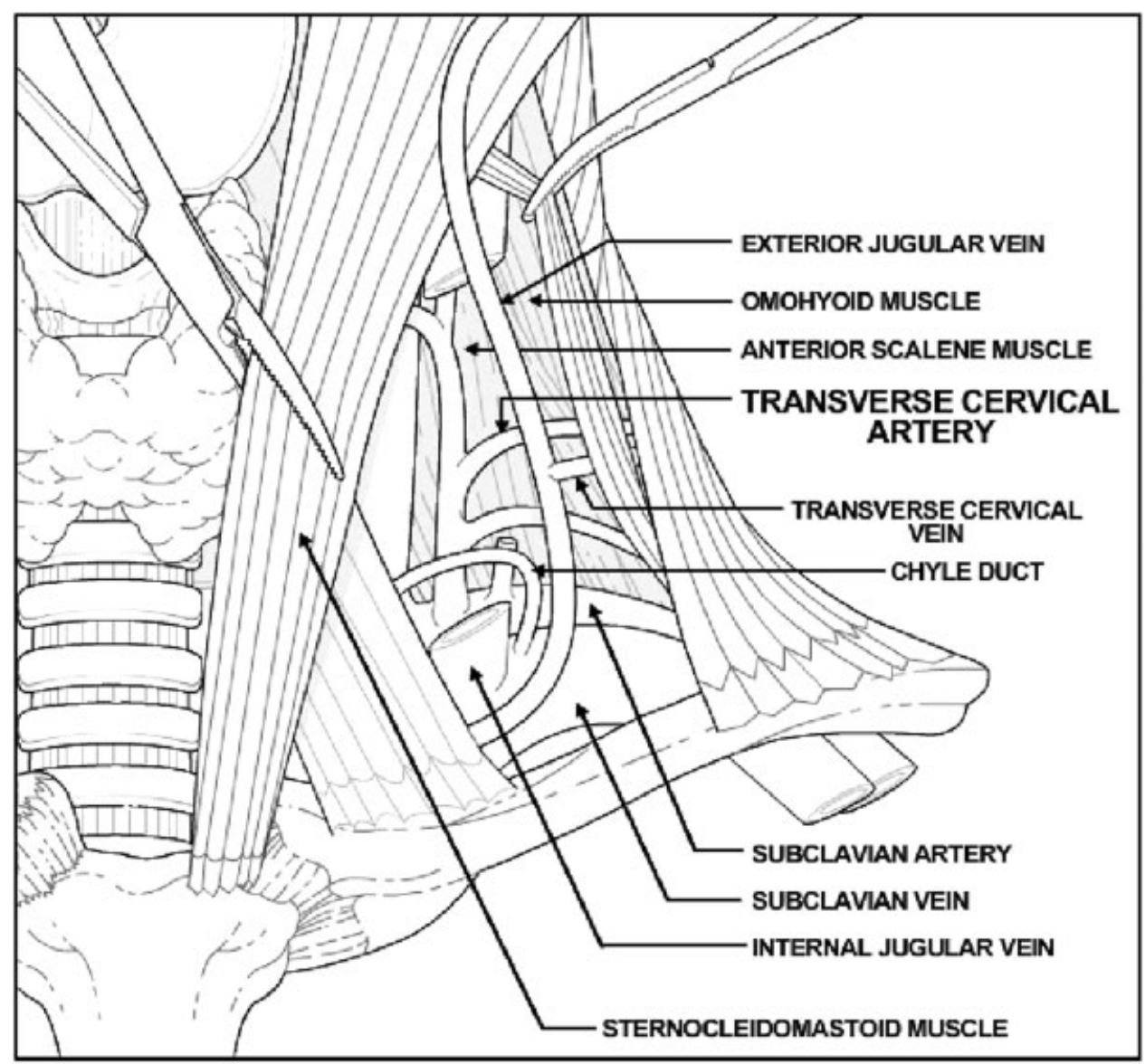

Fig. 1 Illustration of the left transverse cervical artery for anastomosis with the omohyoid muscle retracted laterally.

not prevent the reconstructive surgeon from dissecting the left side. Extensive blunt dissection or overzealous sharp dissection should be avoided and meticulous attention to the presence of chyle during a forced Valsalva will decrease the likelihood of an iatrogenic fistula. In this review, $25 \%$ of reconstruction were performed using the left TCA without chylous complications. However, if chyle is visualized, the chyle duct should be explored and ligated with a surgical clip or suture ligation. ${ }^{10}$ While microvascular repair of the chyle duct has been described, this is challenging with a high risk of postoperative complications and the authors recommend against this approach. The right TCA is exposed similarly and is less likely to disturb the chyle system. The TCA can be traced distally several centimeters to provide increased length and increased vessel diameter.

\section{Microvascular Reconstruction Using the TCA}

Our experience with the TCA system has shown several advantages of using this as the recipient vessel ( - Table $\mathbf{1}$ ). Favorable pedicle geometry is critical to avoid acute pedicle angles and inadvertent kinking. The transverse cervical vessels are oriented in the longitudinal direction and provide a favorable geometry for oral cavity, laryngeal, and pharyngeal reconstruction. ${ }^{11}$ For long pedicle lengths, such as the radial forearm in oral cavity defects, the TCA provides an ideal geometric alignment for the microvascular anastomosis. This is especially true when the antecubital vein that bridges both the cephalic vein and the venae comitantes of the radial artery is chosen for the venous anastomosis. This can be anastomosed to either the TCV or the external jugular vein in the level $\mathrm{V}$ with a straight pedicle drainage pattern.

Table 1 Comparison of TCA versus ECA for microvascular anastomosis

\begin{tabular}{|l|l|l|}
\hline Comparison Criteria & TCA & ECA \\
\hline Radiation field exposure & Outside high-dose radiation field & Inside high-dose radiation field \\
\hline Size $(\mathrm{mm})$ & 3 & $2-4$ \\
\hline Head rotation & Less impact on geometry & Increased impact on geometry \\
\hline Favorable defect location & Pharynx, larynx, oral cavity & Midface, pharynx, larynx, oral cavity, \\
\hline Unfavorable defect location & Mandible, midface, scalp, osseous & Scalp \\
\hline
\end{tabular}

Abbreviations: ECA, external carotid artery; TCA, transverse cervical artery. 
Furthermore, the relatively long pedicle length between the defect and anastomosis reduces the effect of head movement on vessel geometry, which reduces the chance of vessel kinking with head rotation. An additional benefit of the TCA related to its location low in the neck is that the mandible does not restrict the operating surgeon's field. This enables a more relaxed hand position for microvascular anastomosis. This is especially useful for less experienced microsurgeon.

The most notable advantage of the TCA is in the subset of patients previously treated with radiation or surgical therapy. The anatomical position of the TCA in the inferior neck and deep to the clavicles is associated with a relatively lower dose of radiation therapy compared with branches of the ECA. ${ }^{12}$ The inferior location of the TCA therefore can act as a salvage recipient vessel when branches of the ECA are no longer suitable for microsurgical anastomosis either due to radiation induced damage to the intima or from previous surgical compromise. It is our practice in these salvage cases to plan for the TCA from the outset of the surgical case. If the reconstruction is midline, then the right TCA is explored first either through the neck dissection incision or through a separate $4 \mathrm{~cm}$ in the anatomical triangle described above. If the right TCA is not suitable, we proceeded to explore the left TCA. A noted limitation of the TCA is the smaller vessel caliber compared with branches of the external carotid artery. Nevertheless, this is overcome using traditional anastomosis techniques to address vessel mismatch. An additional limitation is the longer pedicle length required, particularly for oral cavity or midface reconstruction, to reach the position of the TCA in the inferior neck. This can be partially overcome by tracing the TCA distally to obtain greater length.

\section{Conclusion}

In advanced or recurrent head and neck cancer, especially in the setting of prior oncologic therapy, free tissue transfer is an essential component of the reconstruction to reconstitute form and function. However, finding an appropriate and healthy recipient artery or vein is often very challenging due to the extent of disease and the presence of prior therapy in terms of radiation, surgery, or a combination thereof. The anatomical location of the TCA is such that it is less likely to be compromised from prior comprehensive neck dissections or be involved by disease. Furthermore, as the TCA is relatively shielded by the clavicles, it is less likely to be exposed to high dose of radiation. Therefore, the transverse cervical vessels make an ideal receipt vessel. This series illustrates the technique for using TCA, demonstrating that the transverse cervical vessels can be readily and successfully applied to complex head and neck reconstruction particularly in a salvage setting.

\section{Note}

The authors would like to thank Tim Grant for preparing the figure.

\section{Conflict of Interest}

None declared.

\section{References}

1 Slaughter DP, Southwick HW, Smejkal W. Field cancerization in oral stratified squamous epithelium; clinical implications of multicentric origin. Cancer 1953;6(05):963-968

2 Guelinckx PJ, Boeckx WD, Fossion E, Gruwez JA. Scanning electron microscopy of irradiated recipient blood vessels in head and neck free flaps. Plast Reconstr Surg 1984;74(02):217-226

3 Xu ZF, Duan WY, Zhang EJ, et al. Transverse cervical vessels as recipient vessels in oral and maxillofacial microsurgical reconstruction after former operations with or without radiotherapy. World J Surg Oncol 2015;13:183

4 Ciudad P, Agko M, Manrique OJ, et al. The retrograde transverse cervical artery as a recipient vessel for free tissue transfer in complex head and neck reconstruction with a vessel-depleted neck. Microsurgery 2017;37(08):902-909

5 Weiglein AH, Moriggl B, Schalk C, Künzel KH, Müller U. Arteries in the posterior cervical triangle in man. Clin Anat 2005;18(08): 553-557

6 Lamberty BG. The supra-clavicular axial patterned flap. Br J Plast Surg 1979;32(03):207-212

7 Daseler EH, Anson BJ. Surgical anatomy of the subclavian artery and its branches. Surg Gynecol Obstet 1959;108(02):149-174

8 Goodwin WJ Jr, Rosenberg GJ. Venous drainage of the lateral trapezius musculocutaneous island flap. Arch Otolaryngol 1982; 108(07):411-413

$9 \mathrm{Yu}$ P. The transverse cervical vessels as recipient vessels for previously treated head and neck cancer patients. Plast Reconstr Surg 2005;115(05):1253-1258

10 Crumley RL, Smith JD. Postoperative chylous fistula prevention and management. Laryngoscope 1976;86(06):804-813

11 Urken ML, Vickery C, Weinberg H, Buchbinder D, Biller HF. Geometry of the vascular pedicle in free tissue transfers to the head and neck. Arch Otolaryngol Head Neck Surg 1989;115(08): 954-960

12 Urken ML. Recipient vessel selection in free tissue transfer to the Head and Neck. In: Urken ML, Cheney ML, Blackwell KE, Harris JR, Hadlock TA, Futran N, eds. Atlas of Regional and Free Flaps for Head and Neck Reconstruction. Baltimore, MA: Lippincott Williams and Wilkins; 2012:505-520 